\title{
Procedure volume is one determinant of centre effect in mechanically ventilated
} patients

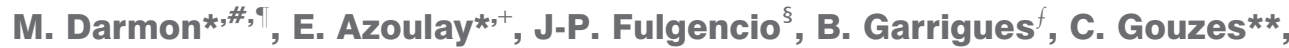 \\ P. Moine ${ }^{\# \#}$, D. Villers ${ }^{\top \uparrow}$, V. Teboul*, J-R. le Gall**, and S. Chevret ${ }^{+,++, \S \S}$
}

ABSTRACT: Survival rates vary significantly between intensive care units, most notably in patients requiring mechanical ventilation (MV). The present study sought to estimate the effect of hospital MV volume on hospital mortality.

We included 179,197 consecutive patients who received mechanical ventilation in 294 hospitals. Multivariate logistic regression models with random intercepts were used to estimate the effect of annual MV volume in each hospital, adjusting for differences in severity of illness and case mix.

Median annual MV volume was 162 patients (interquartile range 99-282). Hospital mortality in MV patients was $31.4 \%$ overall, $40.8 \%$ in the lowest annual volume quartile and $28.2 \%$ in the highest quartile. After adjustment for severity of illness, age, diagnosis and organ failure, higher MV volume was associated with significantly lower hospital mortality among MV patients (OR 0.9985 per 10 additional patients, $95 \% \mathrm{Cl} 0.9978-0.9992 ; p=0.0001)$. A significant centre effect on hospital mortality persisted after adjustment for volume effect $(p<0.0001)$.

Our study demonstrated higher hospital MV volume to be independently associated with increased survival among MV patients. Significant differences in outcomes persisted between centres after adjustment for hospital MV volume, supporting a role for other significant determinants of the centre effect.

KEYWORDS: Acute respiratory distress syndrome, databases, factual, intensive care unit, outcome assessment, performance, quality of healthcare

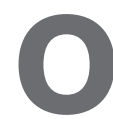

ver the last two decades, advances in the evaluation of healthcare outcomes have shed light on the determinants of survival in patients undergoing various medical and surgical procedures [1-5]. Variations in outcomes are generally believed to stem from differences between institutions, patient characteristics, case mix or organisational factors [3, 4, 6, 7]. Among the factors that lead to differences between institutions (the centre effect), procedure volume, defined as the number of patients receiving a specific procedure in the hospital each year, has been identified as playing a major role [8-12].

Identifying the determinants of the centre effect may suggest means of improving the quality of care [4]. For instance, staffing differences contribute to the centre effect, and the Leapfrog Group has estimated that applying intensive care unit (ICU) physician staffing standards might save $>54,000$ lives $\cdot \mathrm{yr}^{-1}$ [13]. Thus, comparing quantitative performance across institutions may help us to understand how structure and care processes affect patient survival [1, 14, 15].

Acute respiratory failure is common in patients admitted to the ICU [16]. Despite the advances achieved over the last decade, survival has not substantially improved among patients who receive mechanical ventilation (MV) [16, 17]. This fact suggests a need for updating guidelines for MV, and improving the quality and process of care in patients receiving MV. In a large study of 37 acute care hospitals in the USA, the annual volume of patients receiving $\mathrm{MV}$ was a major determinant of survival among these patients [11]. However, other studies found no association between volume and outcome, suggesting a need for additional work on larger numbers of patients and hospitals [18-20].

The purpose of this study was to examine the relationship between the number of critically ill
AFFILIATIONS

*Medical Intensive Care Unit,

+"Biostatistics Dept,

§s UMRS 717 Inserm, Saint-Louis

University Hospital,

${ }^{\S}$ Surgical Intensive Care Unit, Tenon University Hospital, Assistance

Publique des Hôpitaux de Paris,

+Paris-7 University, Paris,

\#Medical Intensive Care Unit,

"Thrombosis Research Group, EA

3065, Saint-Etienne University

Hospital and Saint-Etienne Medical School, Saint-Etienne,

${ }^{f}$ Medical-Surgical Intensive Care Unit, Pays D'Aix Hospital, Aix-enProvence,

**Medical Intensive Care Unit, Ales Hospital, Ales, and

"Medical-Surgical Intensive Care Unit, Nantes-Hotel Dieu University Hospital, Nantes, France.

\#\# Dept of Anesthesiology, Health Sciences Centre, University of Colorado at Denver, Denver, CO, USA.

CORRESPONDENCE

M. Darmon

Medical-Surgical Intensive Care Unit

Saint-Etienne University Hospital

Avenue Albert Raimond

42270 Saint-Priest-en-Jarez

Saint-Etienne

France

E-mail: michael.darmon@

chu-st-etienne.fr

Received:

Dec 102009

Accepted after revision:

June 142010

First published online:

July 012010

Online ISSN 1399-3003 
patients receiving MV (volume) and hospital survival of those patients. The study included 179,197 consecutive patients who received MV over a 2-yr period in the 294 ICUs in the French Ministry of Health (Paris, France) database.

\section{PATIENTS AND METHODS Source of data}

We used the French nationwide database maintained by the Ministry of Health to obtain standardised hospital discharge data on all in-patient stays in public and private hospitals. The standardised forms used to feed the database collect administrative, demographic and medical information on the patients, including dates and type of admission and discharge (and, therefore, length of hospital stay), vital status at hospital discharge, diagnoses classified using the International Classification of Diseases, 10th revision (ICD-10-CM), and procedures classified using the French Medical Procedures Catalogue. The primary diagnosis, either presented at admission or occurring during the hospital stay, is defined as the diagnosis that accounted for the largest part of the care provided during the hospital stay. This information is used to classify the hospital stay using the French equivalent of the diagnosis-related group (DRG) system (Programme de Médicalisation des Systèmes d'Information). For patients admitted to ICUs, severity of illness, as measured by the Simplified Acute Physiology Score II (SAPSII), was recorded [21].

Because the Ministry of Health database contains standardised administrative and medical data on all consecutive hospital discharges, it can be used to derive national estimates. However, the order in which diagnoses are made and procedures used are not recorded in the database. To assess the validity of the database information for assessing ICU admissions and diagnoses, we conducted a comparison with data from the medical records of 6,652 patients in five hospitals [22]. Differences in selected in-patient stays occurred for only $0.2 \%$ of stays and were chiefly related to patients who were admitted then given MV outside the ICU.

\section{Study populations}

The main study population comprised adults ( $\geqslant 18$ yrs of age) who were discharged from a French public hospital in 2004 or 2005, and who had a SAPSII value $>0$ and a record of receiving conventional $\mathrm{MV}$, indicating that they were very probably admitted to the ICU. Patients who received only continuous positive airway pressure or noninvasive MV were not included. Hospitals that reported $<100$ admissions $\cdot \mathrm{yr}^{-1}$, no deaths over the year and fewer than five MV patients per year were excluded from the analysis. The final dataset consisted of 179,197 patients with MV, in 294 hospitals.

To evaluate the impact of the underlying disease requiring $\mathrm{MV}$, we defined three other study populations, based on ICD10-CM codes: acute respiratory distress syndrome (ARDS), cardiogenic shock and intoxications (Table S1 in the supplementary material). These are important groups of low- to highrisk medical conditions. Although clinically relevant, patients with sepsis, severe sepsis or septic shock were not considered in this subgroup analysis, as a consequence of the complex coding system for sepsis in the ICD-10-CM (several codes) and the impossibility of adequately distinguishing hospital- from community-acquired sepsis in the chosen database.

\section{Outcome measures}

The exposure variable was annual hospital volume, defined as the number of patients who received MV each year in each hospital. Since we studied 294 hospitals over 2 yrs, we had 588 hospital-year units, which were studied separately, since volume in a given hospital may vary from year to year. The main outcome measure was hospital mortality.

\section{Statistical analysis}

Statistical analysis was performed by S. Chevret (Biostatistics Depart, Saint-Louis University Hospital and UMRS 717 Inserm, Paris, France).

Descriptive data are presented as median (interquartile range $(\mathrm{IQR})$ ). To evaluate the effects of covariates on the binary outcome variable hospital death, we used logistic regression models with random intercepts for each hospital, introducing

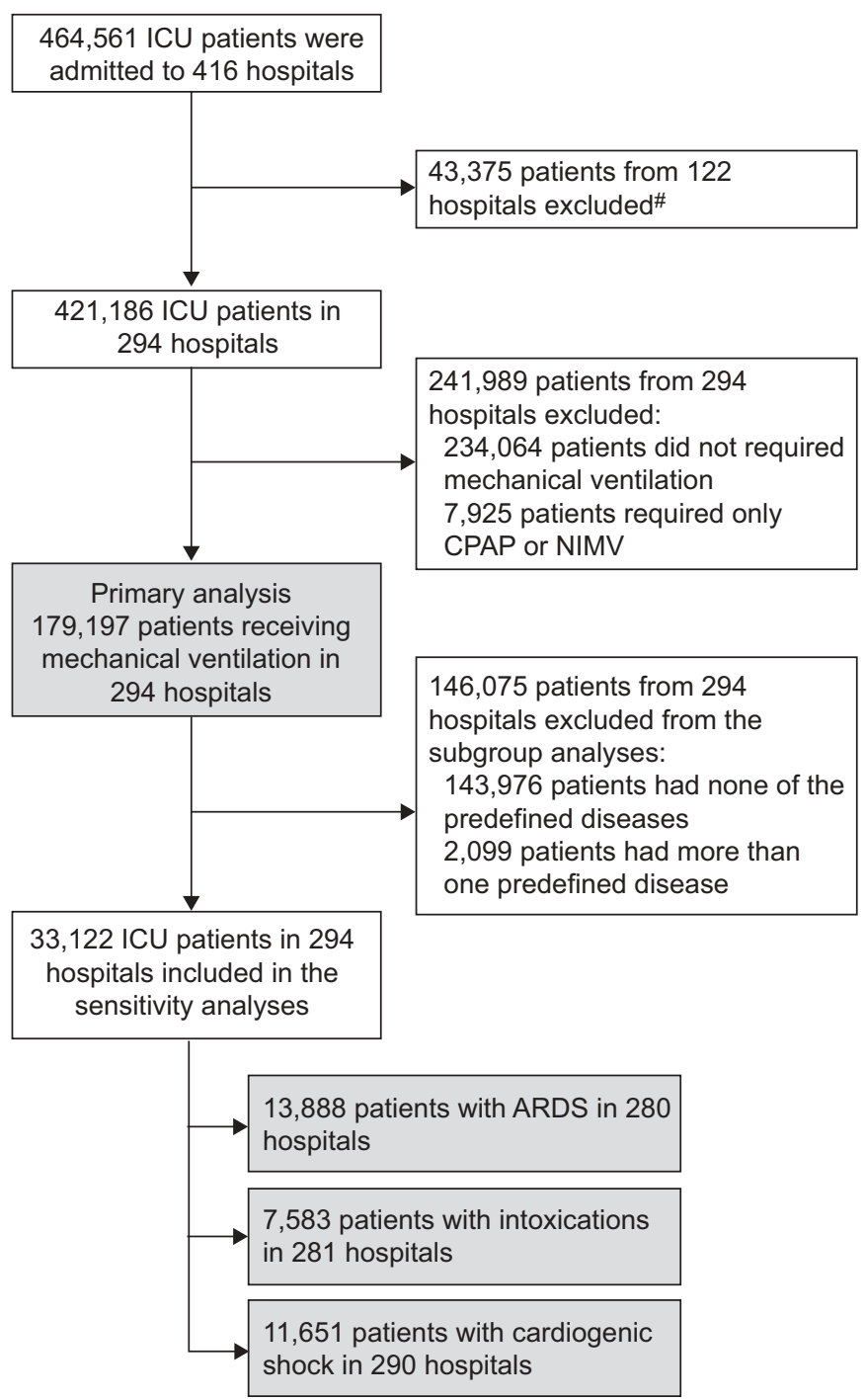

FIGURE 1. Flow chart of patients and hospitals in the study. Shaded boxes represent populations of patients included in the study. ICU: intensive care unit; CPAP: continuous positive airway pressure; NIMV: noninvasive mechanical ventilation; ARDS: acute respiratory distress syndrome. "\#: reasons for exclusion were $<100$ admissions $\cdot y r^{-1}$ and fewer than five mechanically ventilated patients per year. 
hospital volume as a continuous variable. We addressed potential confounding due to case-mix variations by controlling for severity of illness and additional variables related to ICU survival in multivariate models. Severity of illness was assessed based on the SAPSII (with higher scores indicating greater severity of illness and higher risk of death) on the day of admission [21]. To adjust for case mix, secondary analyses were planned in the three study populations (ARDS, cardiogenic shock and intoxications). Given the large sample sizes, only p-values of $\leqslant 0.001$ were considered significant.

Statistical regression models were built using the glmmML function of $\mathrm{R}$ to estimate the maximum likelihood via numerical integration with the aide of Gauss-Hermite quadrature [23].

\section{RESULTS}

In 2004 and 2005, 421,186 patients were admitted to ICUs in the 294 hospitals, and among them, 179,197 patients received MV. Figure 1 shows a flow chart of the patients and hospitals in the study. Median hospital volume was $162 \mathrm{MV}$ patients $\cdot$ year $^{-1}$ (IQR 99-282).

\section{Patient characteristics according to hospital volume}

The characteristics of the 179,197 MV patients according to hospital volume are shown in table 1 . Median age was 63 yrs (IQR 49-74 yrs) and 111,819 (62.4\%) patients were male. Medical DRGs accounted for $57.6 \%$ of the hospital stays. In addition to MV, 80,489 (44.9\%) patients required vasoactive agents and 16,933 (9.4\%) patients required renal replacement therapy. Median length of hospital stay was 14 days (IQR 6-28 days). Median SAPSII at admission was 43 (IQR 29-59), indicating a predicted in-hospital mortality rate of $30.6 \%$ (IQR 9.7-66.1\%); the observed hospital mortality rate was $31.4 \%$. Reasons for ICU admission and severity of illness evaluated by SAPSII value varied widely with hospital volume. Median SAPSII was lower in high- than in low-volume hospitals $(43.7 \pm 22.2$ in the highest volume quartile versus $51.3 \pm 22.6$ in the lowest volume quartile; $\mathrm{p}<0.0001$ ). This difference in severity of illness probably stemmed, in part, from two other

\section{TABLE 1 Patient characteristics by hospital volume quartile}

Characteristics
Hospital volume patients $\cdot \mathrm{yr}^{-1}$

\begin{tabular}{llll}
\hline & $99-162$ & $163-282$ & 282
\end{tabular}

\begin{tabular}{|c|c|c|c|}
\hline 146 & 148 & 147 & 147 \\
\hline $68.2 \pm 21.4$ & $129.9 \pm 19.7$ & $210.7 \pm 32.6$ & $809.8 \pm 681.3$ \\
\hline 9965 & 19220 & 30969 & 119043 \\
\hline 8836 (88.7) & 17054 (88.7) & 26665 (86.1) & 102653 (86.2) \\
\hline $64.6 \pm 17.4$ & $64.0 \pm 17.2$ & $61.8 \pm 17.5$ & $59.0 \pm 17.5$ \\
\hline $5753(57.7)$ & $11440(59.5)$ & $19004(61.4)$ & 75995 (63.8) \\
\hline $51.3 \pm 22.6$ & $50.5 \pm 21.6$ & $51.6 \pm 22.2$ & $43.7 \pm 22.2$ \\
\hline 5517 (55.4) & 10331 (53.7) & $16682(53.9)$ & $43174(36.3)$ \\
\hline $2772(27.8)$ & $5540(28.8)$ & 8634 (27.9) & 59051 (49.6) \\
\hline $749(7.5)$ & 2065 (10.7) & $2807(9.1)$ & 10109 (8.5) \\
\hline 1900 (19.1) & 4699 (24.4) & 7320 (23.6) & 23389 (19.6) \\
\hline 1497 (15.0) & 3753 (19.5) & 6659 (21.5) & 18788 (15.8) \\
\hline 632 (6.3) & $1532(8.0)$ & $2344(7.6)$ & 8976 (7.5) \\
\hline $592(5.9)$ & $1198(6.2)$ & 2037 (6.6) & 4302 (3.6) \\
\hline 290 (2.9) & 577 (3.0) & 1091 (3.5) & 3374 (2.8) \\
\hline $85(0.8)$ & $171(0.9)$ & $312(1.0)$ & $661(0.6)$ \\
\hline $120(1.2)$ & $273(1.4)$ & 461 (1.5) & $1394(1.2)$ \\
\hline $4073(40.9)$ & 8864 (46.1) & $14910(48.1)$ & $52642(44.2)$ \\
\hline 376 (3.8) & $1548(8.0)$ & 3192 (10.3) & 11817 (9.9) \\
\hline 1805 (18.1) & 3667 (19.1) & 7507 (24.2) & 25801 (21.7) \\
\hline 1520 (15.2) & 3791 (19.7) & 6926 (22.4) & 22678 (19.0) \\
\hline $19.2 \pm 24.2$ & $22.1 \pm 27.2$ & $21.5 \pm 27.7$ & $22.4 \pm 28.8$ \\
\hline $4062(40.8)$ & 7347 (38.2) & $11356(36.7)$ & 33607 (28.2) \\
\hline
\end{tabular}

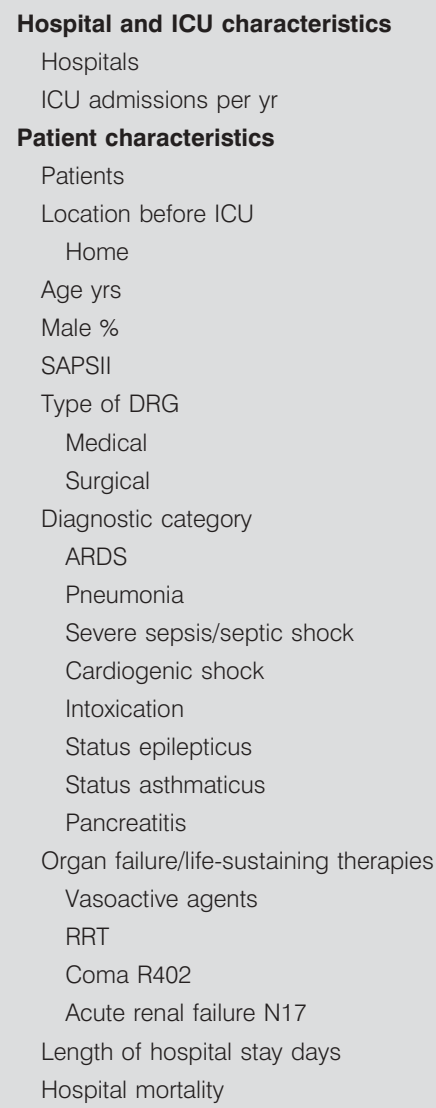


characteristics of high-volume hospitals, namely, younger patient age $(59 \pm 17.5$ versus $64.6 \pm 17.4$ yrs in the highest and lowest quartiles, respectively; $\mathrm{p}<0.0001$ ) and larger proportion of patients with surgical DRGs (49.6 versus $27.8 \%$; $\mathrm{p}<0.0001$ ). In high-volume hospitals, there were proportionately more patients requiring vasoactive agents ( 44.2 versus $40.9 \%$; $p<0.0001$ ) and /or renal replacement therapy (9.9 versus 3.8\%; $<<0.0001$ ), and patients with coma (21.7 versus $18.1 \%$; $\mathrm{p}<0.0001)$.

\section{Patients with predefined conditions}

Of the 179,197 MV patients, 33,122 (18.5\%) patients had one of the three predefined conditions: 13,888 patients had ARDS, 11,651 patients had cardiogenic shock and 7,583 patients had intoxications. The prevalence of these three conditions varied widely between annual volume quartiles (table 1). The conditionspecific annual volume (defined as the number of patients with each condition in each study hospital in each study year) was 14 patients $\cdot$ hospital ${ }^{-1} \cdot \mathrm{yr}^{-1}$ (IQR 6-30 patients $\cdot$ hospital $^{-1} \cdot \mathrm{yr}^{-1}$ ) for ARDS, 10 patients $\cdot$ hospital $^{-1} \cdot \mathrm{yr}^{-1}$ (IQR 5-20 patients $\cdot$ hospital $^{-1} \cdot \mathrm{yr}^{-1}$ ) for cardiogenic shock and 10 patients $\cdot$ hospital ${ }^{-1} \cdot \mathrm{yr}^{-1}$ (IQR 4-17 patients $\cdot$ hospital ${ }^{-1} \cdot \mathrm{yr}^{-1}$ ) for intoxications. Hospital mortality was $43.1 \%$ in the 33,122 patients overall, $53.7 \%$ in patients with ARDS, $59.7 \%$ in patients with cardiogenic shock and $2.2 \%$ in patients with intoxications.

\section{Impact of hospital volume on outcome}

Unadjusted hospital mortality decreased from each volume quartile to the next, from $40.8 \%$ in the lowest volume hospitals to $28.2 \%$ in the highest (table 1). After adjusting for potential confounders (severity of illness, age, DRG, diagnoses during the ICU stay, organ failures and life-sustaining treatments) and

\begin{tabular}{|c|c|c|c|}
\hline TABLE 2 & \multicolumn{3}{|c|}{$\begin{array}{l}\text { Factors associated with hospital mortality: } \\
\text { results of the multivariate mixed regression } \\
\text { model }\end{array}$} \\
\hline Parameter & & OR (95\% Cl) & p-value \\
\hline \multicolumn{2}{|l|}{ Intercept } & $0.0126(0.011-0.014)$ & $<0.0001$ \\
\hline \multicolumn{2}{|c|}{ Hospital volume per 10 patients $\cdot \mathrm{yr}^{-1}$} & $0.9985(0.9978-0.9992)$ & 0.0001 \\
\hline \multicolumn{2}{|c|}{ Age per yr } & $1.02(1.018-1.020)$ & $<0.0001$ \\
\hline \multicolumn{2}{|l|}{ Female sex } & $0.90(0.88-0.93)$ & $<0.0001$ \\
\hline \multicolumn{2}{|c|}{ SAPS II per point } & $1.047(1.046-1.0475)$ & $<0.0001$ \\
\hline \multicolumn{2}{|c|}{ Surgical DRG } & $0.46(0.44-0.47)$ & $<0.0001$ \\
\hline \multicolumn{2}{|c|}{ ARDS } & $1.65(1.58-1.72)$ & $<0.0001$ \\
\hline \multicolumn{2}{|l|}{ Pneumonia } & $0.82(0.80-0.84)$ & $<0.0001$ \\
\hline \multicolumn{2}{|c|}{ Severe sepsis } & $1.18(1.14-1.22)$ & $<0.0001$ \\
\hline \multicolumn{2}{|c|}{ Cardiogenic shock } & $1.35(1.29-1.41)$ & $<0.0001$ \\
\hline \multicolumn{2}{|c|}{ Intoxication } & $0.07(0.06-0.08)$ & $<0.0001$ \\
\hline \multicolumn{2}{|c|}{ Status epilepticus } & $0.65(0.60-0.70)$ & $<0.0001$ \\
\hline \multicolumn{2}{|c|}{ Status asthmaticus } & $0.63(0.54-0.74)$ & $<0.0001$ \\
\hline \multicolumn{2}{|c|}{ Pancreatitis } & $0.92(0.83-1.03)$ & 0.1346 \\
\hline \multicolumn{2}{|c|}{ Vasoactive agents } & $2.41(2.34-2.48)$ & $<0.0001$ \\
\hline \multicolumn{2}{|c|}{ RRT } & $1.79(1.71-1.87)$ & $<0.0001$ \\
\hline \multicolumn{2}{|l|}{ Coma } & $1.71(1.66-1.77)$ & $<0.0001$ \\
\hline \multicolumn{2}{|c|}{ Acute renal failure } & $1.31(1.26-1.36)$ & $<0.0001$ \\
\hline
\end{tabular}

incorporating a random hospital effect, each hospital volume increase of 10 patients year $^{-1}$ was associated with a significant decrease in hospital mortality (OR 0.9985, 95\% CI 0.99780.9992; $\mathrm{p}=0.0001$ ) (table 2). Figures 2 and 3 show the effect of hospital volume on hospital mortality, evaluated as a continuous variable. The multivariate mixed regression model showed persistence of a significant centre effect $(p<0.0001)$.

To assess the sensitivity of our findings, we repeated the analysis with varying assumptions about the patient population (table 3). Our results were not affected by the exclusion of outlier hospitals. Nevertheless, total annual hospital volume was no longer associated with hospital mortality when we excluded patients in surgical DRGs (OR 0.9992 per 10 patients. $\mathrm{yr}^{-1}, 95 \%$ CI 0.9984-1.0000; $\mathrm{p}=0.0502$ ) or when we adjusted for case mix by restricting our analysis to the patients having any of the three predefined conditions (OR 1.0012 per 10 patients. $\mathrm{yr}^{-1}, 95 \%$ CI 0.999-1.002; $\mathrm{p}=0.06$ ) (table 3).

\section{Impact of hospital volume on outcome of patients with predefined conditions}

Although total annual volume (i.e. number of MV patients per year in each study hospital) was not associated with adjusted hospital mortality, the condition-specific annual volumes for ARDS and cardiogenic shock (i.e. number of patients with each condition per year in each study hospital) was strongly associated with adjusted hospital mortality after adjustment for confounders (table 3). Similarly, the specific hospital random effect correlated positively with the overall hospital random effect in all the populations except the population with intoxications (Figure S2 in the supplementary material).

\section{DISCUSSION}

We used data collected over 2 yrs in the French Ministry of Health database to examine the effect of hospital volume on hospital mortality of patients requiring MV. In a population of $>170,000 \mathrm{MV}$ patients, hospital volume of MV patients correlated negatively with hospital mortality after adjustment for potential confounders. Interestingly, adjustment for this volume effect did not abolish the centre effect on hospital

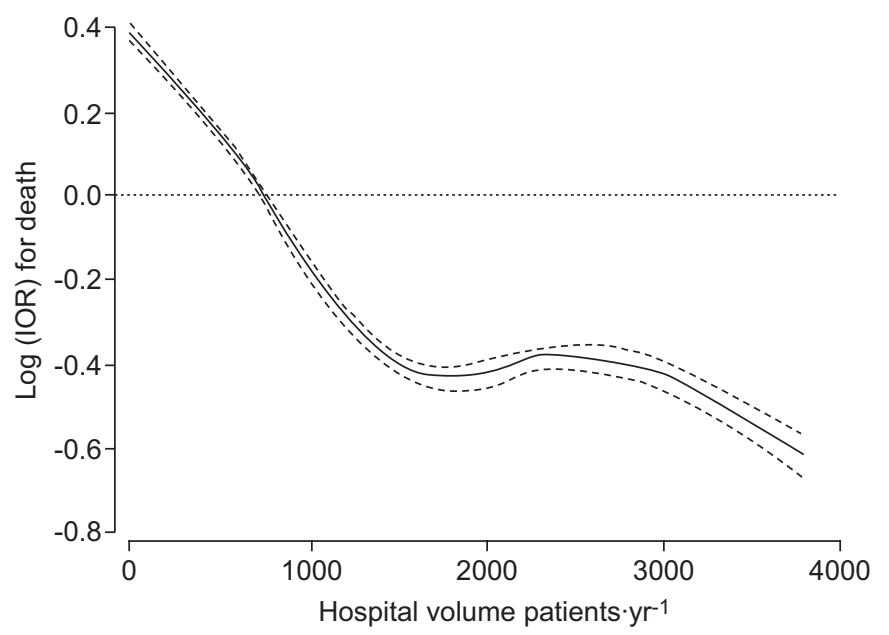

FIGURE 2. Relationship between hospital volume and hospital mortality —: interval OR (IOR); ---: 95\% Cl. 

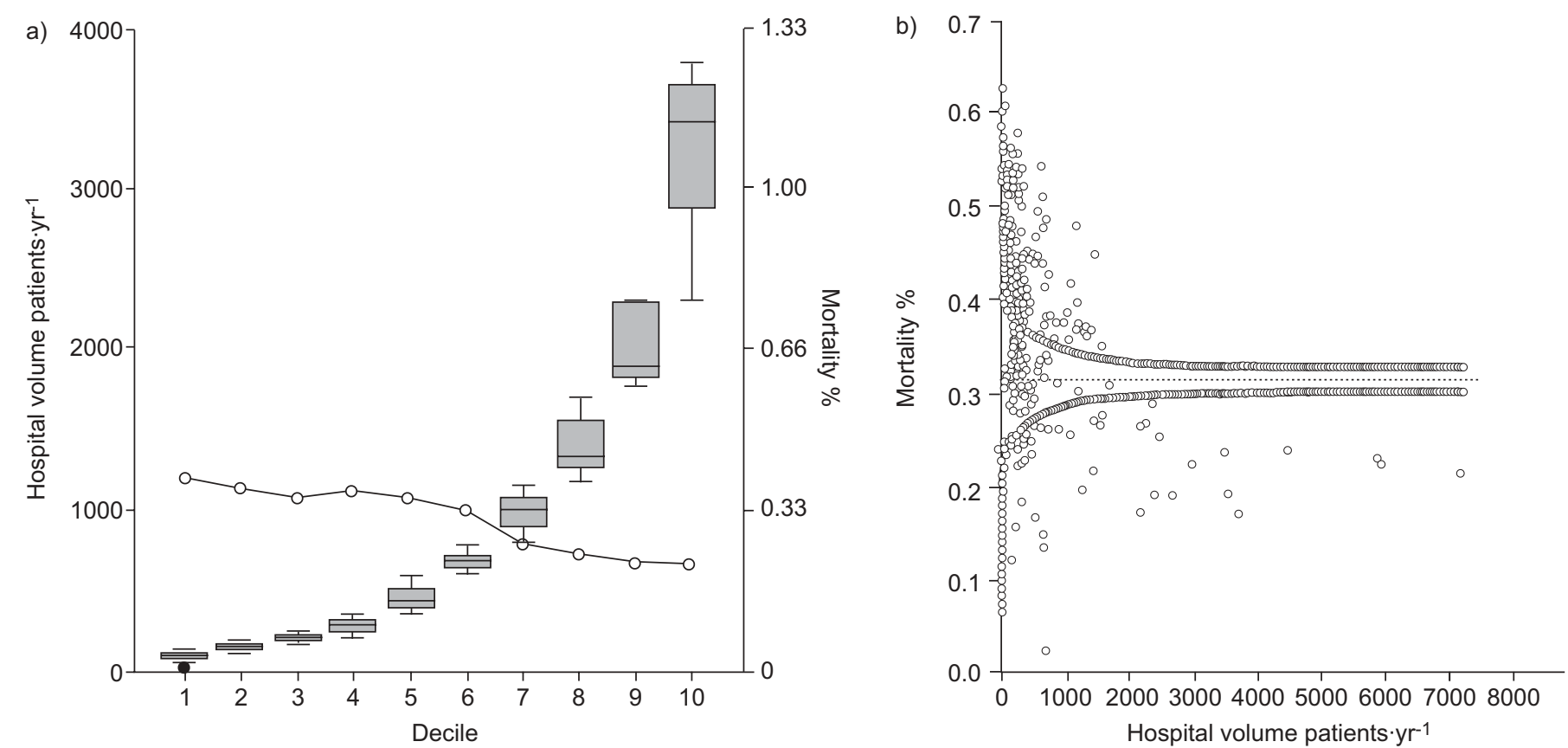

FIGURE 3. Hospital mortality against hospital volume. a) Box plots of annual hospital volumes segregated into ten deciles, with associated hospital mortality $(\bigcirc)$. Boxes represented the interquartile range and whiskers respresent the 5th and 95th percentiles. -: median; $\bullet$ : outliers. b) Funnel plot of hospital mortality.

mortality, indicating a role for other factors that remain to be identified.

The association found in our study between higher volume and higher survival, after controlling for confounders, suggests that the greater clinical experience acquired by managing many patients may translate into higher quality of care. Earlier studies found associations between higher volume and better outcomes in several surgical conditions $[8,9,12]$. Conflicting data have been obtained regarding the volume-outcome association in critical care patients, including medical patients, surgical patients, paediatric patients and neonates $[2,10,11,20$, 24-27]. Several factors may explain these discrepancies. Some of the studies in critically ill patients included small numbers of hospitals [19, 23] and/or patients [20, 24, 28]. Since the effect of hospital volume is usually modest, failure to detect such an effect may indicate inadequate statistical power, especially when adjusting for a cluster effect [29]. In addition, several of these studies were performed in selected ICUs, which may have introduced selection bias [19, 28]. Finally, clustering of the data was disregarded in some studies $[19,28]$.

Another interesting finding from our study is the impact of condition-specific hospital volumes (i.e. annual hospital volume of MV patients with a specific condition), even in the absence of an impact of total hospital MV volume. In sensitivity analyses involving various assumptions about patient populations, after adjustment for confounders, condition-specific hospital volumes were independently associated with hospital mortality. This finding suggests that, even when greater experience with MV has no effect on outcome, greater experience with a specific condition may benefit the patients. Few studies have evaluated the impact of hospital volume of specific diseases. In one study, even an increase of 1 patient $\cdot \mathrm{yr}^{-1}$ having a haematological malignancy and acute respiratory failure was associated with a significant decrease in mortality [25]. Similarly, in a study of ICU admissions from 1991 to 1997, high-volume hospitals had lower mortality rates in medical ICU patients with gastrointestinal diseases (hazard ratio $0.68,95 \%$ CI $0.51-0.85$ ) but not in patients with respiratory or neurological reasons for admission [24]. However, each of the groups in this study included a heterogeneous array of conditions, ranging, for instance, from ARDS to chronic obstructive pulmonary disease in the respiratory group, and from metabolic coma to intracerebral haemorrhage in the neurological group. This heterogeneity may explain the absence of associations between volume and mortality [24]. Our results suggest that centres having extensive experience with specific diagnoses may produce the best outcomes. This result suggests that regionalisation of care and patient referral to high-volume centres would be beneficial, at least when dealing with these specific conditions. However, we found no association between hospital volume and mortality in the subgroup of patients with intoxications; the low hospital mortality of $2.2 \%$ in this subgroup should be taken into account when interpreting this negative result.

Our study has several limitations. First, the information available on the institutions was limited and we were, therefore, unable to adjust mortality for specific characteristics of the centres. However, the use of a multivariate mixed regression model allowed us to incorporate and test for a random cluster effect of the institutions. Patient referral practices were not evaluated in this study. They may affect the impact of volume on mortality, as well as the performance of healthcare institutions [10, 20, 24]. However, they were probably taken into account as part of the centre effect identified in our study, suggesting that hospital volume is associated with outcome independently of the institutions' characteristics. Another limit arises from the database used. 


\section{TABLE 3 Sensitivity analysis for impact of hospital volume on mortality rate}

\begin{tabular}{|c|c|c|c|}
\hline Base model & 179197 & $0.9985(0.9978-0.9992)$ & 0.0001 \\
\hline Excluding patients with surgical conditions & 103200 & & \\
\hline Effect of total annual volume & & $0.9992(0.9984-1.0000)$ & 0.0502 \\
\hline Effect of annual volume of patients with medical condition & & $0.9999(0.9997-1.0000)$ & 0.0244 \\
\hline Effect of total annual volume & & $1.0012(0.9999-1.0025)$ & 0.066 \\
\hline Effect of annual volume for all three conditions & & $0.9987(0.9980-0.9994)$ & 0.0003 \\
\hline ARDS & 13888 & & \\
\hline Effect of total annual volume & & $1.0005(0.9994-1.0017)$ & 0.36 \\
\hline Effect of ARDS annual volume & & $0.9983(0.9973-0.9993)$ & 0.0010 \\
\hline Effect of total annual volume & & $1.0027(0.9998-1.0055)$ & 0.0656 \\
\hline Effect of intoxication annual volume & & $0.9962(0.9883-1.0042)$ & 0.35 \\
\hline
\end{tabular}

Indeed, we used a database that contains information on all patients discharged from healthcare institutions in France. This database is designed to serve as a tool for cost assessments, as opposed to medical research. Data quality and validity may be problematic in databases that are not specifically designed for research. For example, several pieces of information regarding duration of organ support were lacking. Moreover, misleading coding may have affected DRG for the included patients. Nevertheless, for analysis of hospital volume influence in specific conditions, we choose DRG, for which coding was expected to be robust, as a way to limit this bias. In addition, earlier work showed that administrative databases performed relatively well compared to clinical databases, being capable of identifying outlying hospitals with slight [30] or major [31] differences. Finally, our findings may not apply to other countries. In France, most ICUs have high staffing levels [32] compared to other countries [13, 32]. Nevertheless, our study provides robust information on the impact of hospital volume on hospital mortality in countries with high ICU staffing levels. Our findings support the practice of regionalisation in these countries.

In conclusion, our study, the largest to date in this field, showed that higher hospital volume was associated with lower adjusted hospital mortality among patients requiring MV in a healthcare system characterised by high-level ICU staffing. In addition, condition-specific hospital volumes (i.e. annual hospital volume of MV patients having a specific condition) were also associated with hospital mortality in patients with ARDS or cardiogenic shock. Our findings support studies of the regionalisation of care, as well as patient referral to highvolume centres, at least when dealing with these specific conditions. Lastly, the centre effect persisted after adjustment for hospital volume. Therefore, further studies are needed to look for structural or organisational factors associated with this residual centre effect.

\section{SUPPORT STATEMENT}

The study was supported by a grant from the Assistance PubliqueHôpitaux de Paris (PHRC AOM04056), a nonprofit, governmentfunded organisation.

\section{STATEMENT OF INTEREST}

None declared.

\section{REFERENCES}

1 Li TC, Phillips MC, Shaw L, et al. On-site physician staffing in a community hospital intensive care unit. Impact on test and procedure use and on patient outcome. JAMA 1984; 252: 2023-2027.

2 Pollack MM, Katz RW, Ruttimann UE, et al. Improving the outcome and efficiency of intensive care: the impact of an intensivist. Crit Care Med 1988; 16: 11-17.

3 Pronovost PJ, Angus DC, Dorman T, et al. Physician staffing patterns and clinical outcomes in critically ill patients: a systematic review. JAMA 2002; 288: 2151-2162.

4 Pronovost PJ, Jenckes MW, Dorman T, et al. Organizational characteristics of intensive care units related to outcomes of abdominal aortic surgery. JAMA 1999; 281: 1310-1317.

5 Treggiari MM, Martin DP, Yanez ND, et al. Effect of intensive care unit organizational model and structure on outcomes in patients with acute lung injury. Am J Respir Crit Care Med 2007; 176: 685-690.

6 Brilli RJ, Spevetz A, Branson RD, et al. Critical care delivery in the intensive care unit: defining clinical roles and the best practice model. Crit Care Med 2001; 29: 2007-2019.

7 Carson SS, Stocking C, Podsadecki T, et al. Effects of organizational change in the medical intensive care unit of a teaching hospital: 
a comparison of "open" and "closed" formats. JAMA 1996; 276: 322-328.

8 Begg CB, Cramer LD, Hoskins WJ, et al. Impact of hospital volume on operative mortality for major cancer surgery. JAMA 1998; 280: 1747-1751.

9 Birkmeyer JD, Siewers AE, Finlayson EV, et al. Hospital volume and surgical mortality in the United States. N Engl J Med 2002; 346: 1128-1137.

10 Kahn JM, Goss CH, Heagerty PJ, et al. Hospital volume and the outcomes of mechanical ventilation. N Engl J Med 2006; 355: 41-50.

11 Kahn JM, Linde-Zwirble WT, Wunsch H, et al. Potential value of regionalized intensive care for mechanically ventilated medical patients. Am J Respir Crit Care Med 2008; 177: 285-291.

12 Urbach DR, Baxter NN. Does it matter what a hospital is "high volume" for? Specificity of hospital volume-outcome associations for surgical procedures: analysis of administrative data. Qual Saf Health Care 2004; 13: 379-383.

13 Angus DC, Shorr AF, White A, et al. Critical care delivery in the United States: distribution of services and compliance with Leapfrog recommendations. Crit Care Med 2006; 34: 1016-1024.

14 Brown JJ, Sullivan G. Effect on ICU mortality of a full-time critical care specialist. Chest 1989; 96: 127-129.

15 Pollack MM, Ruttimann UE, Getson PR. Pediatric risk of mortality (PRISM) score. Crit Care Med 1988; 16: 1110-1116.

16 Esteban A, Ferguson ND, Meade MO, et al. Evolution of mechanical ventilation in response to clinical research. Am J Respir Crit Care Med 2008; 177: 170-177.

17 Phua J, Badia JR, Adhikari NK, et al. Has mortality from acute respiratory distress syndrome decreased over time? A systematic review. Am J Respir Crit Care Med 2009; 179: 220-227.

18 Clark DE, DeLorenzo MA, Lucas FL, et al. Initial presentation of older injured patients to high-volume hospitals is not associated with lower 30-day mortality in Medicare data. Crit Care Med 2007; 35: 1829-1836.

19 Jones J, Rowan K. Is there a relationship between the volume of work carried out in intensive care and its outcome? Int J Technol Assess Health Care 1995; 11: 762-769.

20 Needham DM, Bronskill SE, Rothwell DM, et al. Hospital volume and mortality for mechanical ventilation of medical and surgical patients: a population-based analysis using administrative data. Crit Care Med 2006; 34: 2349-2354.

21 Le Gall JR, Lemeshow S, Saulnier F. A new Simplified Acute Physiology Score (SAPS II) based on a European/North American multicenter study. JAMA 1993; 270: 2957-2963.

22 De Coster C, Quan H, Finlayson A, et al. Identifying priorities in methodological research using ICD-9-CM and ICD-10 administrative data: report from an international consortium. BMC Health Serv Res 2006; 6: 77.

23 R Project. Package "glmmML". http://cran.r-project.org/web/ packages/glmmML/glmmML.pdf Date last accessed: November 18, 2010. Date last updated: October 13, 2009.

24 Durairaj L, Torner JC, Chrischilles EA, et al. Hospital volumeoutcome relationships among medical admissions to ICUs. Chest 2005; 128: 1682-1689.

25 Lecuyer L, Chevret S, Guidet B, et al. Case volume and mortality in haematological patients with acute respiratory failure. Eur Respir J 2008; 32: 748-754.

26 Marcin JP, Romano PS. Impact of between-hospital volume and within-hospital volume on mortality and readmission rates for trauma patients in California. Crit Care Med 2004; 32: 1477-1483.

27 Tilford JM, Simpson PM, Green JW, et al. Volume-outcome relationships in pediatric intensive care units. Pediatrics 2000; 106: 289-294.

28 Iapichino G, Gattinoni L, Radrizzani D, et al. Volume of activity and occupancy rate in intensive care units. Association with mortality. Intensive Care Med 2004; 30: 290-297.

29 Panageas KS, Schrag D, Riedel E, et al. The effect of clustering of outcomes on the association of procedure volume and surgical outcomes. Ann Intern Med 2003; 139: 658-665.

30 Parker JP, Li Z, Damberg CL, et al. Administrative versus clinical data for coronary artery bypass graft surgery report cards: the view from California. Med Care 2006; 44: 687-695.

31 Glance LG, Li Y, Osler TM, et al. Impact of patient volume on the mortality rate of adult intensive care unit patients. Crit Care Med 2006; 34: 1925-1934.

32 Wunsch H, Angus DC, Harrison DA, et al. Variation in critical care services across North America and Western Europe. Crit Care Med 2008; 36: 2787-2793. 